\title{
Organization of psychiatric care for persons of gerontological age at the outpatient stage
}

\section{Valentina Lebedeva, Irina Kupriyanova, Olga Pavlova Mental Health Research Institute, Tomsk National Research Medical Center, Russain Academy of Sciences, Tomsk, Russia}

Objective: to study need for psychiatric gerontological care in primary care unit.

Background: Relevance of the problem of mental disorders in elderly persons is determined by high prevalence, difficulty of therapeutic support and is caused by comorbidity with somatic pathology.

Material and Methods: The sample included 221 (male $27.5 \%$, female $-72.5 \%$ ) patients of general medicine network aged above 60 years. Clinical-dynamic, clinicalpsychopathological, screening-questionnaire, clinical-follow-up, statistical methods were used.

Results and Discussion: Patients were diagnosed with mental disorders according to ICD-10 as follows:

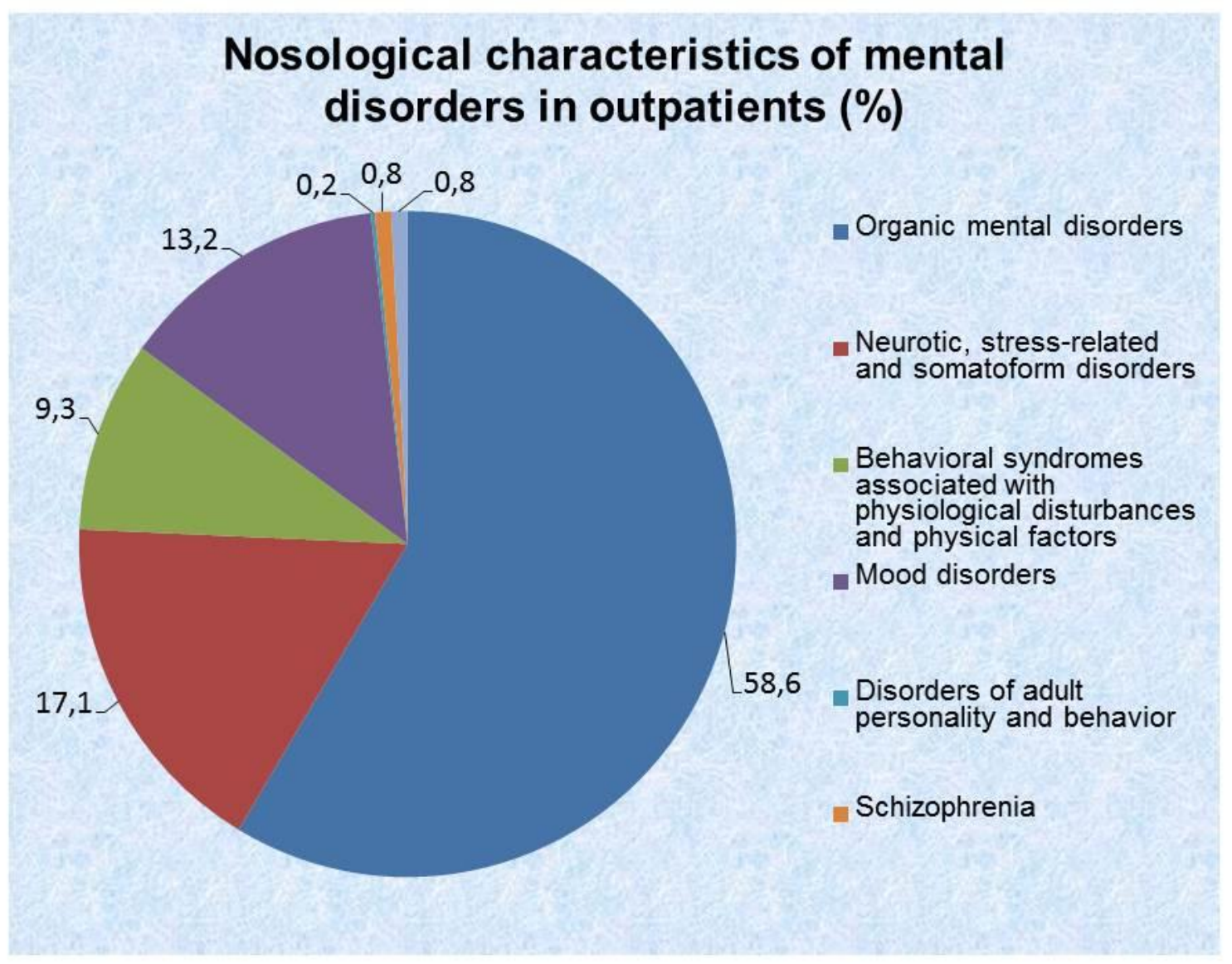

Depending on severity of the state and mental pathology three main groups of patients in need for consultative-diagnostic and therapeutic assistance of a psychiatrist were distinguished:

Group I - risk group - patients with acute somatogenias with transient psychotic equivalents;

Group II - patients in need for psychiatric consultative assistance on request;

Group III - patients in need for permanent consultative psychiatric assistance and regular course therapy.

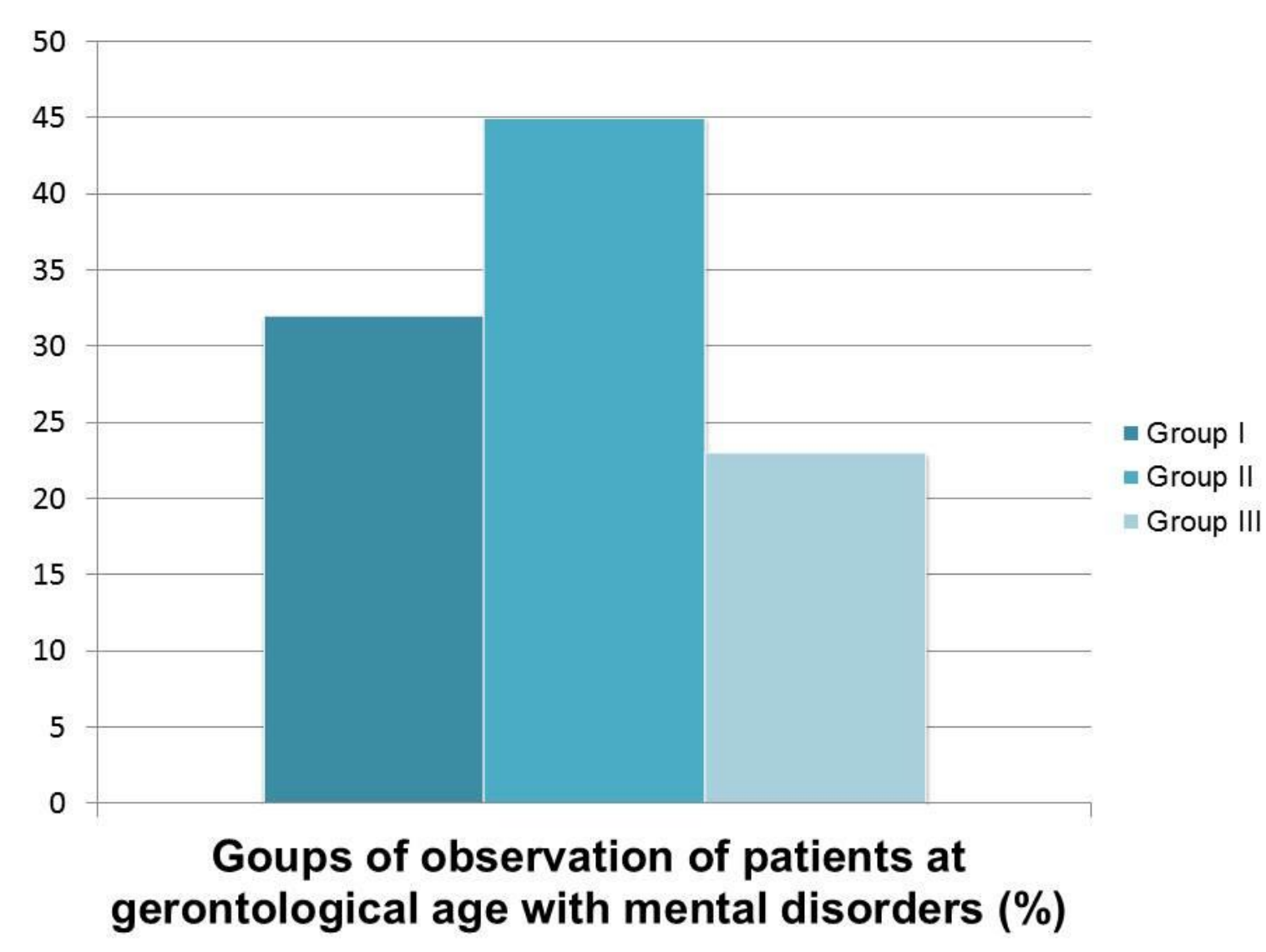

Syndrome aspect of the problem in gerontological patients with combined mental and somatic pathology is of diagnostic and rehabilitative-prognostic significance. Mental state qualified within the leading syndrome was the main target of the therapy.

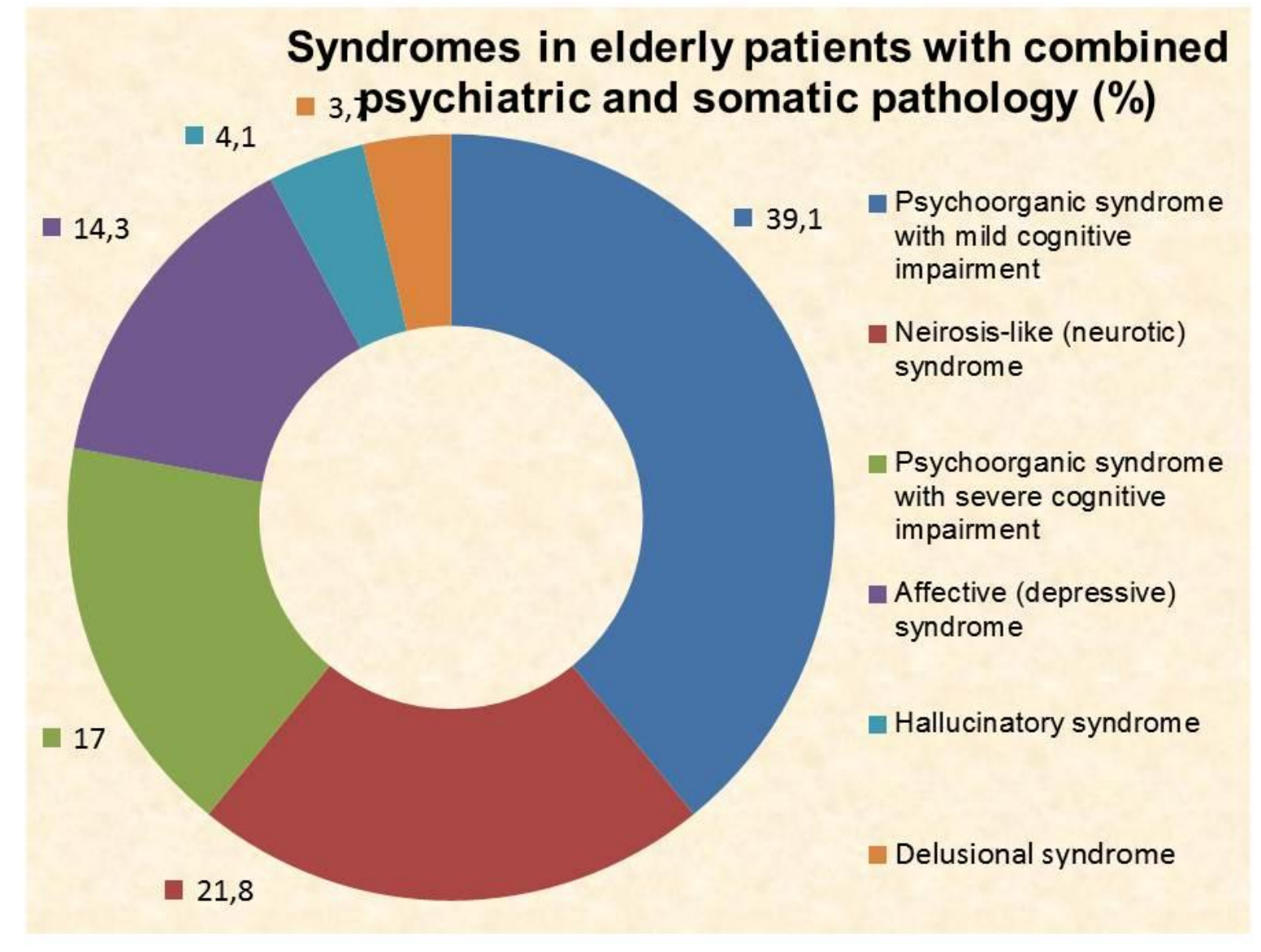

Therapeutic interventions were based on the principle of complexity, consistency, continuity of therapeutic activities. This complex included psychotherapeutic, psychopharmacotherapeutic, psychocorrective methods, measures directed at improvement of family functioning in patients of gerontological age.

At stage $I$ of the treatment against the background of somatotropic, general health-improving, nootropic therapy the choice of psychotropic drug was performed. Treatment was initiated with small doses which were heightened gradually up to therapeutically needed dose.

Stage /I of basic therapy was directed at achievement of positive effect. Treatment at this stage lasted 2-4 months (neurotic disorders, psychogenically caused depressions, emotional-unstable disorders and mild cognitive impairments of organic origin. More long-term period (6-10 months) of treatment was in patients with anxiety, melancholic depressions, organic disorders.

Stage III of maintenance therapy varied from several months to 305 and more years - follow-up of patients after a course of inpatient treatment.

When assessing the results of the treatment of elderly people a positive dynamics was observed in $78 \%$, without dynamics $17.5 \%$, worsening of the state $-4.5 \%$.

Thus, psychohygienic service at a somatic polyclinic allows not only providing assistance to patients with borderline mental disorders but also detects among elderly patients the risk group for development of psychotic disorders typical for later life, 